Original Research Paper

\title{
Rice Grain Moisture Determination Using Microstrip Wide- Ring and Microstrip Coupled-Line Sensors
}

\author{
${ }^{1}$ Hou Kit Mun, ${ }^{2}$ Kok Yeow You and ${ }^{2}$ Mohamad Ngasri Dimon \\ ${ }^{I}$ Department of Electrical and Electronic Engineering, \\ Faculty of Engineering and Information Technology, Southern University College, Malaysia \\ ${ }^{2}$ Department of Radio Communication Engineering, \\ Faculty of Electrical Engineering, Universiti Teknologi Malaysia, Malaysia
}

\author{
Article history \\ Received: 21-04-2014 \\ Revised: 23-06-2014 \\ Accepted: 30-03-2015 \\ Corresponding Author: \\ Kok Yeow You, \\ Department of Radio \\ Communication Engineering, \\ Faculty of Electrical \\ Engineering, Universiti \\ Teknologi Malaysia, Malaysia \\ Email: kyyou@fke.utm.my
}

\begin{abstract}
Determining the Moisture Content (MC) inside rice grain is an important element for grain processesing such as harvesting, storage, quality control and transportation. A microstrip wide-ring sensor and a microstrip coupled-line sensor with low insertion loss were developed to operate at relatively low frequency to determine the $\mathrm{MC}$ and relative complex permittivity of rice grain. The complex permittivity for rice grain with various moisture levels was measured by the proposed sensors based on the resonance technique. Calibration equations for measurement of grain $\mathrm{MC}$ were obtained and validated with white rice for MC ranging from 10 to $28 \%$ wet basis. The coupled-line sensor showed better sensitivity to moisture measurement as compared to the wide-ring sensor. For a $1 \%$ change in $\mathrm{MC}$, the changes of resonant frequency for the wide-ring and coupled-line sensors were 4.36 and $10.69 \mathrm{MHz}$ respectively. Meanwhile, the wide-ring sensor had a higher accuracy in MC prediction than the coupled-line sensor. The average errors in moisture prediction for wide-ring and coupled-line sensors were 0.85 and $1.30 \%$ respectively.
\end{abstract}

Keywords: Moisture Content, Microstrip Coupled-Line, Microstrip WideRing, Rice Grain, Dielectric Constant, Loss Factor

\section{Introduction}

Rice is a main source of nutrition for human beings especially for Asiatic populations. Moisture Content (MC) inside rice grain is a crucial parameter for grain processing such as harvesting, storage, quality control and transportation. For instance, rice grain is usually harvested between 19 and 25\% MC for maximum grain yield and needs to be dried to $14 \%$ or less, depending on the season and the weather, for safe storage. Also, the ideal MC for milling is $14 \%$ in order to maximize the head rice and minimize broken grains. Thus, precise determination of the rice grain $\mathrm{MC}$ is important.

The conventional standard method for MC determination for grain is the oven-drying method. Although this can achieve high precision, it is timeconsuming and impractical. To overcome this, indirect methods such as resistance, RF capacitance and the microwave technique have been developed. Among these, microwave technique is promising as the measurement via microwave frequency is unaffected by the ionic conductivity of water (Meyer and Schilz,
1981). There are several applications reported that used the microwave technique to sense grain $\mathrm{MC}$, such as a microwave microstrip ring resonator for single wheat grain (Abegaonkar et al., 1999) as well as a prototype moisture meter using a microwave transceiver (Kim et al., 2002), multi-layer microstrip moisture sensor (Jafari et al., 2010 ) and cylindrical slot antennas for rice grain quality (You et al., 2011). Besides, microwave technique was also applied in MC determination for oil palm fruit (You et al., 2010a), soybean (Shrestha et al., 2014), tuber (Zainuddin et al., 2013), clay granule (Mextorf et al., 2012), latex (Ansarudin et al., 2012; Mohammadi et al., 2012) and mortar (Jusoh et al., 2011).

Among the microwave techniques, the microstrip ring offers many advantages; for instance, nondestructive measurement, easy sample loading and unloading compared with the waveguide resonant cavity technique (Joshi et al., 1997), easy fabrication into a field-portable device (Sarabandi and Li, 1997), no end effect compared to the single line resonator (Chang and Hsieh, 2004), maximum transmitted power as compared with a rejection filter (Sumesh Sofin and Aiyer, 2005) 
and accurate measurement in dusty environments (Kraszewski, 1998). However, a typical microstrip ring with loose coupling will exhibit a high insertion loss of about $10 \mathrm{~dB}$ at the resonant frequency (Chang and Hsieh, 2004). When a signal is transmitted through the microstrip ring with high insertion loss, the transmitted signal will become very low. Because of this very low transmission signal, some of the low-cost measurement devices are unable to detect the transmitted signal. For this reason, this paper has designed a microstrip ring sensor with low insertion loss characteristic.

In microwave sensing, the relative complex permittivity is an essential parameter to determine MC of grain. It is because the interaction of microwaves with the material is governed by the complex permittivity and the dielectric constant of water is much higher than the dry material (Trabelsi et al., 2009). Complex permittivity measurement techniques can be classified into transmission-reflection and resonance techniques. However, some of the existing microwave grain moisture sensor studies (Joshi et al., 1997; Abegaonkar et al., 1999; You et al., 2011) lacked of complex permittivity characterization with respect to the MC. Limited studies that considered this characterization were based mainly on the transmission technique which has the disadvantage of operating at relatively high frequencies $(9 \mathrm{GHz}$ and 10.5 $\mathrm{GHz}$ ) (Kim et al., 2002; Jafari et al., 2010) with the higher cost of microwave components that operate at high frequency. On the other hand, the resonance technique can accurately measure the low loss material with a low loss factor, compared to the transmission technique (Sheen, 2009). To the best of authors' knowledge, resonancebased microstrip ring sensor has not been applied to rice grain complex permittivity measurement. Moreover, this technique with high insertion loss, will suffer difficulty in resonance extraction due to broadening of the resonance curve when testing material with a high MC.

The microstrip coupled-line has been widely used in filter application. It can be developed as a bandpass filter with low insertion loss within the passband, that can be easily designed at any desired centre frequency. Besides that, it is small in size, light weight and easy to fabricate. A previous study by You et al. (2010b), applied the microstrip coupled-line only to rice powder $\mathrm{MC}$ measurement. In addition, the calibration equation for $\mathrm{MC}$ determination and the complex permittivity measurement were not addressed in that study. There are no studies on applying a microstrip coupled-line to rice grain MC and rice grain complex permittivity measurements.

This paper proposes microstrip wide-ring and microstrip coupled-line sensors for rice grain MC and relative complex permittivity measurements. The microstrip ring was designed to have wide ring to provide a relatively large contact area with rice grains. Also, the $50 \Omega$ feed lines were directly coupled with the ring to realize low insertion loss at the resonant frequency. The low insertion loss characteristic of the sensor will make the resonance measureable even for material with high MC. Bulk grain measurement in this study is distinct from the other single grain measurement (Abegaonkar et al., 1999) as the grain orientation inside the resonator greatly affects the measurement sensitivity and accuracy. Moreover, the proposed coupled-line sensor is able to measure the bulk grain $\mathrm{MC}$ and complex permittivity rather than the powder based MC (You et al., 2010b). Furthermore, a calibration equation of the coupled-line sensor is developed based on the relationship between the $\mathrm{MC}$ and resonant frequency. Both sensors are designed to operate at relatively low frequency $(2.4-2.5 \mathrm{GHz})$ to reduce the cost. The dielectric constant and loss factor of rice grain for difference range of $\mathrm{MC}(10-28 \%)$ are also calculated using the resonance technique via both sensors in this study. Moreover, the effect of various MCs on the physical property of the rice kernels are investigated by using a Scanning Electron Microscope (SEM).

\section{Experiment and Methods}

\section{Fabrication of Sensor}

The microstrip wide-ring and the coupled-line sensors were designed using Microwave Office (MWO) software for an operation frequency range between 1 and $3 \mathrm{GHz}$, where their resonant frequencies are located at 2.49 and $2.45 \mathrm{GHz}$ respectively. All sensors were fabricated on a RT/Duroid 5880 Printed Circuit Board (PCB). The dimensions of the sensors were sketched by using AutoCAD software and printed on transparent paper, then delineated on the substrate by standard photolithography and acid etching. Then, both sensors were held on an aluminum ground with two SMA connectors. Finally, the sensors were covered with an acrylic holder as illustrated in Fig. 1. Figure 2 shows the configuration and dimensions of wide-ring and coupled-line sensors. The dimensions of the sensors are tabulated in Table 1 and 2 .

Table 1. Substrate specifications and dimensions of the widering sensor

\begin{tabular}{llllll}
\hline & \multicolumn{3}{l}{ Dimension $(\mathrm{mm})$} & & \\
Substrate & - & & & \\
specifications & $W_{r}$ & $W_{f}$ & $R_{i}$ & $R_{o}$ & $l$ \\
\hline$\varepsilon_{r, s u b}=2.2$ & 9.03 & 1.14 & 8.92 & 17.96 & 11.09 \\
$\tan \delta=0.001$ & & & & & \\
$h=0.381 \mathrm{~mm}$ & & & & & \\
\hline
\end{tabular}

Table 2. Substrate specifications and dimensions of the coupled-line sensor

\begin{tabular}{lllllllll}
\hline & \multicolumn{1}{l}{ Dimension $(\mathrm{mm})$} & & & & \\
Substrate & -- & - & - & - & & & \\
specifications & $L_{1}$ & $L_{2}$ & $L_{3}$ & $W_{1}$ & $W_{2}$ & $W_{3}$ & $S_{1}$ & $S_{2}$ \\
\hline$\varepsilon_{r, \text { sub }}=2.2$ & 22.3 & 22.1 & 22.0 & 2.4 & 2.0 & 2.3 & 0.3 & 2.0 \\
$\tan \delta=0.001$ & & & & & & & & \\
$h=0.787 \mathrm{~mm}$ & & & & & & & & \\
\hline
\end{tabular}




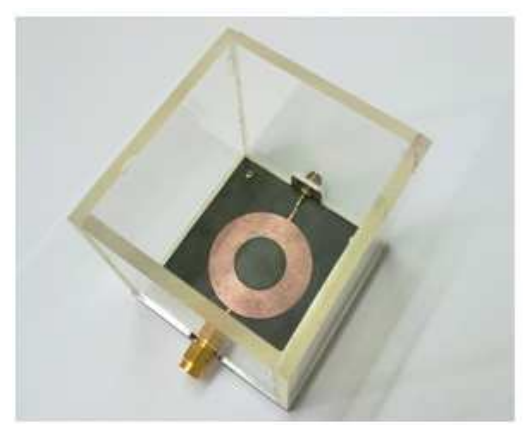

(a)

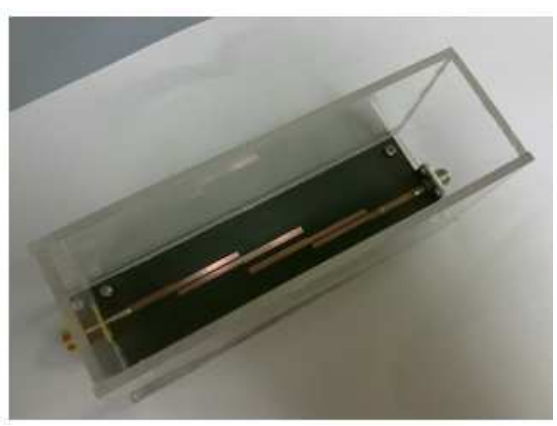

(b)

Fig. 1. The designed sensors (a) wide-ring (b) coupled-line

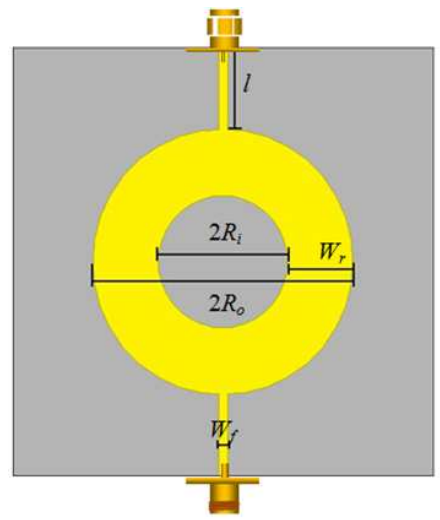

(a)

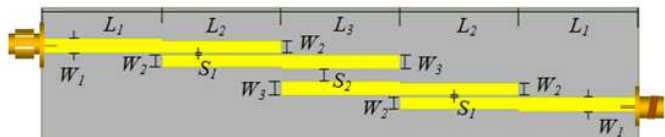

(b)

Fig. 2. Configuration and dimensions of the sensors (a) wide-ring sensor (b) coupled-line sensor

\section{Rice Grain Sample Preparation and Measurement}

$J_{a t i}{ }^{\mathrm{TM}}$ long grain white rice which is grown from the fertile soil of Kedah, the Rice Bowl of Malaysia was used as the experiment sample. The average length, width and MC (wet basis) of the grain were $7.10 \mathrm{~mm}$, $2.04 \mathrm{~mm}$ and $14 \%$ respectively. Rice grain samples with a $\mathrm{MC}$ range from 10 to $28 \%$ were prepared for $\mathrm{MC}$ measurement. The rice grains were divided into different groups with $200 \mathrm{~g}$ per group. To cover the moisture range, some groups of grains were sprayed with different quantities of distilled water to achieve moisture levels ranging from 15 to $28 \%$, while other groups of grains were dried in a forced-convection oven for some period of time to cover the MC range of 10 to $13 \%$. The MC of each rice group was increased gradually from the lowest $\mathrm{MC}$ to the highest by increments of around $1 \%$. For each group, grains were stirred and sealed in a container at $4^{\circ} \mathrm{C}$ for $72 \mathrm{~h}$ to ensure uniform water distribution within the grains. The grains were conditioned to room temperature for $10 \mathrm{~h}$ prior to the measurements.

A full two-port Short-Open-Load-Through (SOLT) calibration was performed at the $50 \Omega$ coaxial cable of the Agilent E5071C network analyzer before being connected to the sensor. After the calibration, grain samples with different level of MC were placed into the sample holder of the sensor. The value of magnitude of transmission coefficient $|T|$, quality factor $Q$ and resonant frequency $f_{r}$ corresponding to different samples were obtained from the network analyzer.

After each measurement, the actual MC of the grains was obtained by the standard oven-drying method. About $10 \mathrm{~g}$ of each sample was dried in a forcedconvection oven at $130^{\circ} \mathrm{C}$ for $24 \mathrm{~h}$ (ASABE, 2006). The $\mathrm{MC}$ in percent was calculated on the wet basis as:

$$
\operatorname{MC}(\mathbf{\%})=\frac{m_{w}}{m_{w}+m_{d}} \times 100
$$

where, $m_{w}$ and $m_{d}$ are the mass of water and dry grain respectively.

\section{Measurement of Dielectric Properties}

\section{Wide-Ring Sensor}

In free space, the particular $f_{r}$ for a microstrip ring depends on the effective dielectric constant $\varepsilon_{\text {eff }}$ and the mean circumference of the ring. For material characterization, the microstrip ring was filled with rice grain that then produced a $f_{r}$ shift and a broadening of the resonance curve compared to free space. The properties of 
the rice grain could be characterized by measuring the shift in $f_{r}$. The $f_{r}$ of the ring sensor can be approximated by:

$$
f_{r}=\frac{n c}{2 \pi r \sqrt{\varepsilon_{e f f}}}
$$

where, $r$ is the mean radius of the ring, $c$ is the speed of light and $n$ is the mode number. The $\varepsilon_{\text {eff }}$ is evaluated as:

$\varepsilon_{e f f}=\frac{\varepsilon_{r, s u b}+\varepsilon_{r}^{\prime}}{2}+\frac{\varepsilon_{r, s u b}-\varepsilon_{r}^{\prime}}{2}\left(1+12 \frac{h}{W_{r}}\right)^{-0.5}$

where, $\varepsilon_{r, s u b}$ is the dielectric constant of the substrate, $W_{r}$ is the width of the ring, $h$ is the substrate thickness and $\varepsilon_{r}{ }^{\prime}$ is the dielectric constant of the material that covers the ring sensor. When the ring sensor is fully filled with air, then $\varepsilon_{r}{ }^{\prime}$ is equal to 1 . In this study, the $50 \Omega$ feed lines were coupled to the ring directly to realize low insertion loss and low radiation loss at the $f_{r}$. Consequently, the coupling effect will affect the $f_{r}$ and Equation 2 was modified as follows:

$$
f_{r}=\chi \frac{n c}{2 \pi r \sqrt{\varepsilon_{e f f}}}
$$

where, $\chi$ is the corrected factor. The value of $\chi$ is obtained through the measured $f_{r}$ when the ring is tested in free space. The value of $\chi$ in this study was 1.0141 . When rice grain with different $\mathrm{MC}$ is placed on the ring sensor, $f_{r}$ will be changed accordingly. The $\varepsilon_{r}{ }^{\prime}$ of the rice grain can be calculated based on the measured $f_{r}$ by using Equations 3 and 4.

Figure 3 shows the current distribution of the ring sensor at $2.49 \mathrm{GHz} f_{r}$. The current at the edge of the coupling point within the feed line and the ring exhibits the coupling effect.

The total loss of the loaded sensor (ring sensor in contact with the rice grains) can be expressed as:

$$
\frac{1}{Q_{l}}=\frac{1}{Q_{u}}+\frac{1}{Q_{d}}+\frac{1}{Q_{r}}
$$

Where:

$Q_{l}=$ The loaded quality factor

$Q_{u}=$ The unloaded (ring sensor in free space) quality factor

$Q_{d}=$ The quality factor due to the dielectric loss

$Q_{r}=$ The quality factor due to the radiation loss

In the present study, the radiation loss was negligible due to the low radiation loss design. Hence, the dielectric loss can be calculated as:

$$
\frac{1}{Q_{d}}=\frac{1}{Q_{l}}-\frac{1}{Q_{u}}
$$

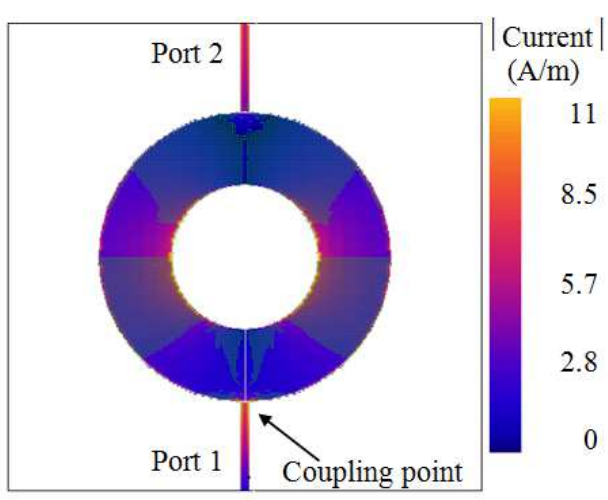

Fig. 3. Current distribution of ring sensor at $2.49 \mathrm{GHz} f_{r}$

The dielectric loss is related directly with the loss $\operatorname{tangent} \tan \delta$ of the loaded material as:

$\frac{1}{Q_{d}}=\tan \delta=\frac{\varepsilon_{r}^{\prime \prime}}{\varepsilon_{r}^{\prime}}$

The loss factor $\varepsilon_{r}{ }^{\prime \prime}$ of the rice grain can be calculated using Equations 6 and 7.

\section{Coupled-Line Sensor}

The coupled-line sensor was designed based on a second order Butterworth bandpass filter principle. For the sake of brevity, the detailed design of the sensor physical dimensions can be referred to in the literature (Gupta et al., 1996; Mongia et al., 2007). The coupledline sensor also exhibits a shift in $f_{r}$ after the sensor is filled with test material. The dielectric constant $\varepsilon_{r}{ }^{\prime}$ and loss factor $\varepsilon_{r}$ " of the rice grain can be determined as follows:

$\varepsilon_{r}^{\prime}=1+2 P \frac{f_{u}-f_{l}}{f_{u}}$

$\varepsilon_{r}^{\prime \prime}=P\left(\frac{1}{Q_{l}}-\frac{1}{Q_{u}}\right)$

where, $P$ is the filling factor, $f_{u}$ and $f_{l}$ represent the unloaded and loaded resonant frequency respectively. The value of $P$ is determined experimentally with a sample of known permittivity. The sample used in this study was corn oil with permittivity obtained by using Agilent 85070E Dielectric Probe and E5071C Network Analyzer. The value of $P$ was determined as 9.0087 .

\section{Results}

\section{Effect of MC on the Physical Property of Rice Kernel}

To study the effect of MCs to the physical property of rice kernels, the surface morphology of rice kernels 
with various moisture levels were investigated. The measurements were performed using a Hitachi TM3000 Tabletop SEM at magnifications of 40 and 1000. Figure 4 illustrates the SEM images for rice kernels with different MCs. As the MC increased, the micro cracks on the surface of the rice kernel could be observed clearly through the microscope. The small cracks were caused by the expansion of the rice kernels.

\section{Variation in Magnitude of Transmission Coefficient with Frequency for Various $M C s$}

Figure 5 illustrates the magnitude of transmission coefficient $|T|$ as a function of frequency corresponding to different MCs for the wide-ring sensor and coupledline sensor. Both sensors exhibited low insertion loss at $f_{r}$ when tested in air. The minimum insertion loss for the wide-ring sensor and coupled-line sensor were close to 0.67 and $1.81 \mathrm{~dB}$ respectively. When the $\mathrm{MC}$ was increased, the $f_{r}$ shifted toward lower frequencies and the maximum value of $|T|$ was decreased accordingly. The decrement of $|T|$ is due to the increment of transmission loss when the grain MC is increased.

\section{Variation in Dielectric Properties with MC}

The relationship between the dielectric constant $\varepsilon_{r}{ }^{\prime}$ and loss factor $\varepsilon_{r}^{\prime \prime}$ for rice grain with various MC percentages is shown in Fig. 6.

The $\varepsilon_{r}{ }^{\prime}$ and $\varepsilon_{r}$ " of rice grain as a function of MC were determined by the wide-ring sensor and coupled-line senor using Equations 3 to 7 and 8 to 9 respectively. Figure 6 shows that both $\varepsilon_{r}{ }^{\prime}$ and $\varepsilon_{r}{ }^{\prime \prime}$ are increased with the grain MC. The average $\varepsilon_{r}{ }^{\prime}$ and $\varepsilon_{r}{ }^{\prime \prime}$ for the 10 to $28 \%$ range of $\mathrm{MC}$ were 2.4 to 3.7 and 0.13 to 0.31 respectively. From (Nelson, 1991), the $\varepsilon_{r}{ }^{\prime}$ and $\varepsilon_{r}{ }^{\prime \prime}$ were 2.33 to 3.46 and 0.26 to 0.54 respectively for the 11 to $20.6 \%$ range of $\mathrm{MC}$.

\section{Variation in MC with Resonant Frequency}

Figure 7 shows the calibration curves relating the different MCs with corresponding resonant frequency $f_{r}$ for both sensors. Calibration equations based on the relationship between the $\mathrm{MC}$ and $f_{r}$ for wide-ring and coupled-line sensor were developed with 25 and 32 sets of rice samples respectively. The calibration equations for the wide-ring sensor and coupled-line sensor are given respectively as:

$$
\begin{aligned}
& M C=-229.28079 f_{r}+566.74913 \\
& M C=-93.52012 f_{l}+218.39002
\end{aligned}
$$

The coefficient of determination $R^{2}$ for Equations 10 and 11 are 0.9272 and 0.8814 respectively.

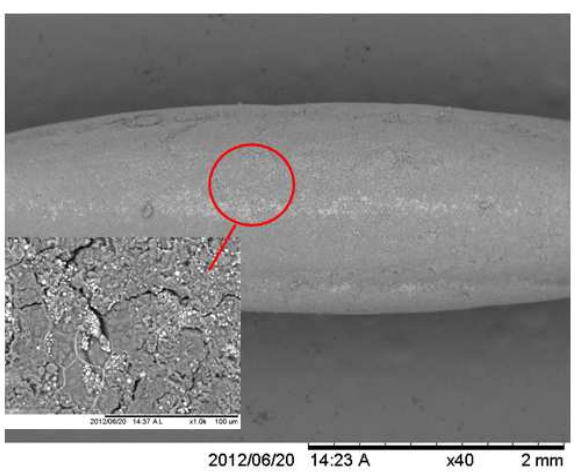

(a)

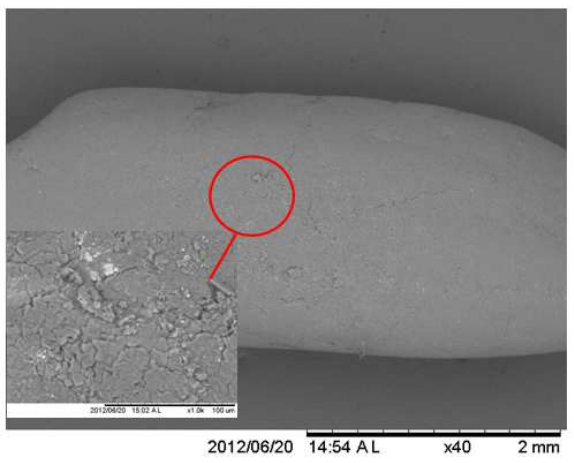

(b)

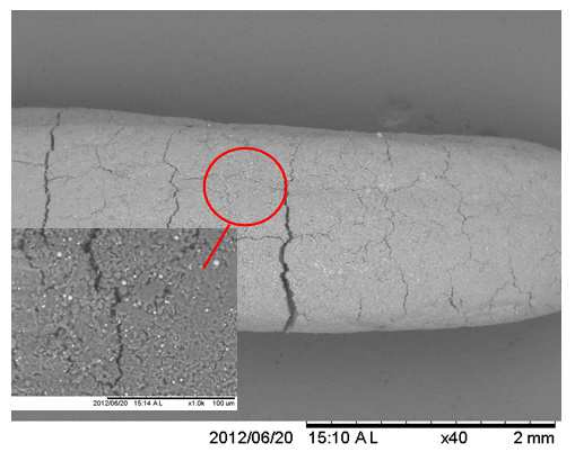

(c)

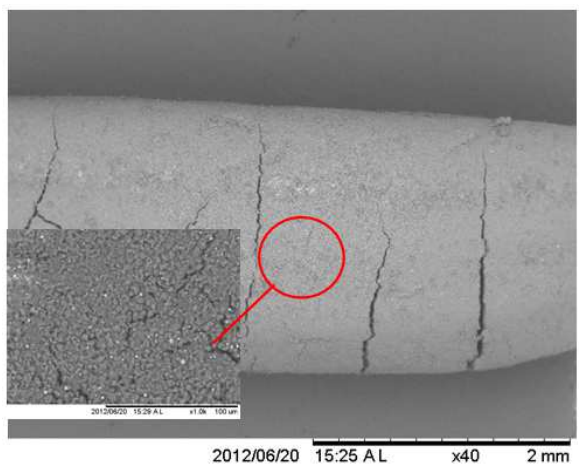

(d)

Fig. 4. SEM images for MC of rice kernels at (a) $10.45 \%$, (b) $14.78 \%$, (c) $20.17 \%$ and (d) $24.97 \%$ at $\times 40$ and $\times 1000$ magnification 


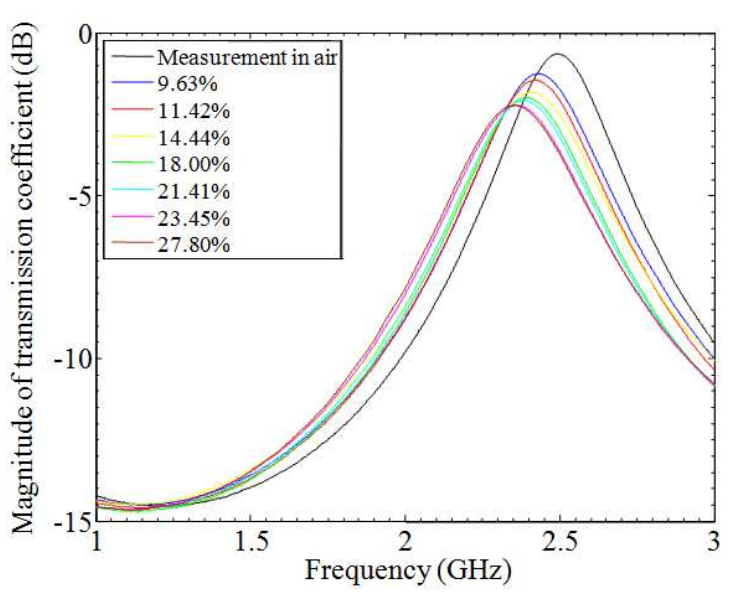

(a)

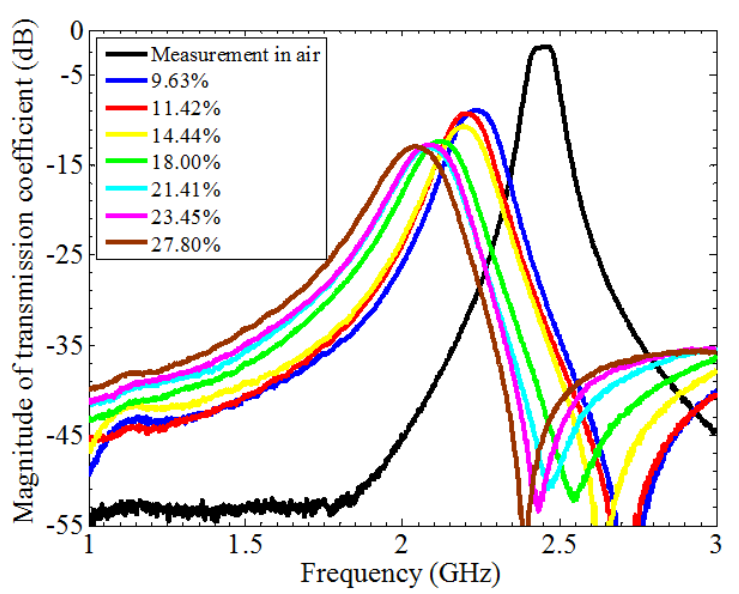

(b)

Fig. 5. Variation in magnitude of transmission coefficient with frequency corresponding to different MCs for (a) the wide-ring sensor (b) the coupled-line sensor

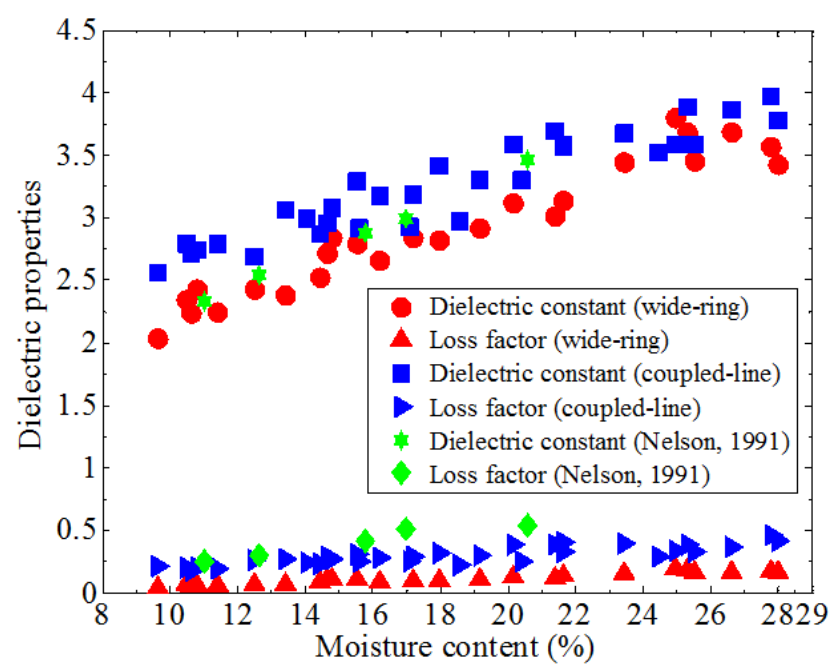

Fig. 6. Variation of dielectric properties with MC

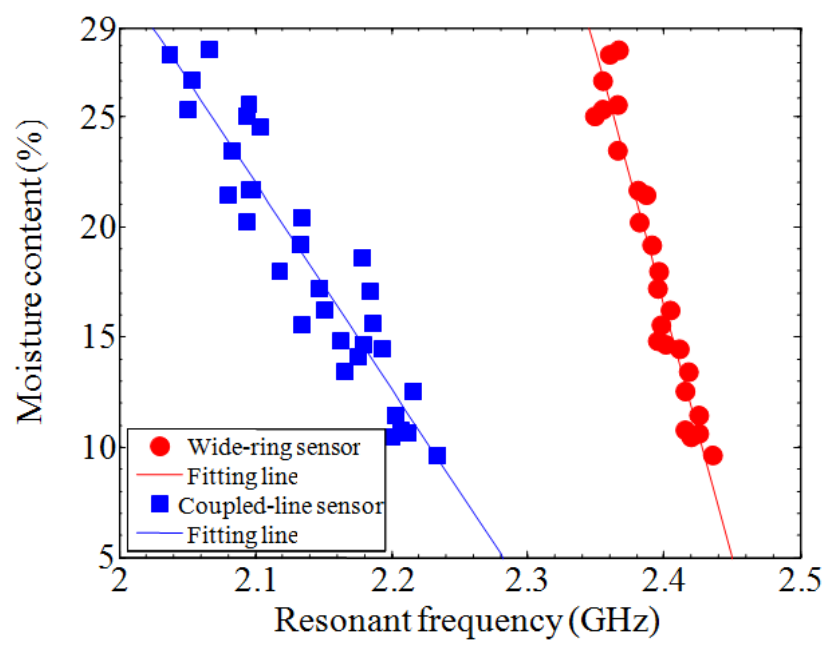

Fig. 7. Variation of MC with resonant frequency 


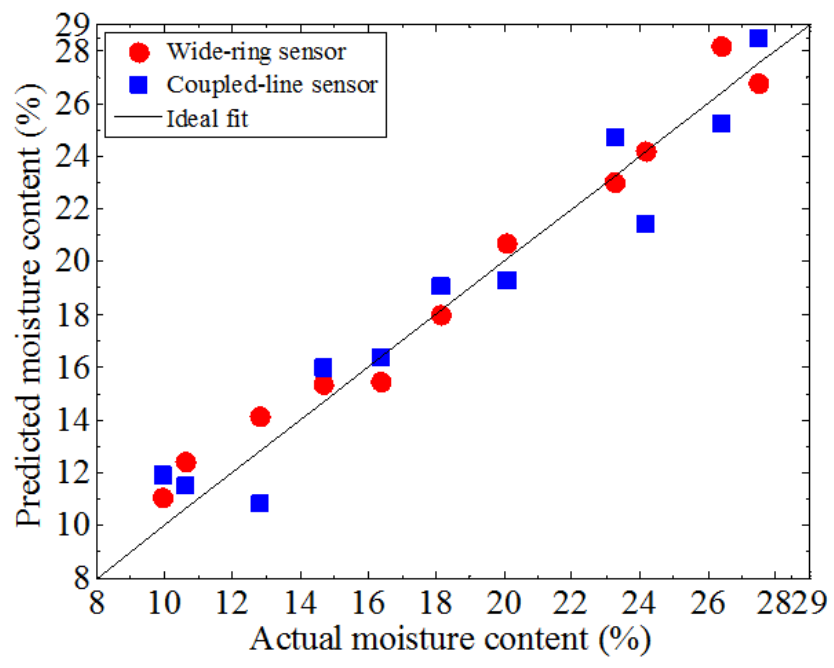

Fig. 8. Predicted MC against actual MC

The $f_{r}$ of both sensors was linear with the change of $\mathrm{MC}$, the $\triangle f \triangle M C$ for wide-ring and coupled-line sensors was $4.36 \mathrm{MHz} / \mathrm{MC}$ and $10.69 \mathrm{MHz} / \mathrm{MC}$ respectively.

\section{The Validation of the Calibration Curves}

Eleven new sets of rice samples over 10 to $28 \%$ of MC were prepared to validate Equations 10 and 11. Figure 8 shows the comparison of the predicted and actual MC for both sensors.

The predicted MCs were calculated from Equations 10 and 11 while the actual MCs were obtained by the standard oven-drying method with the solid line representing the ideal fit. The predicted MCs are close to the line of ideal fit. The $R^{2}$ and average error in MC prediction for the wide-ring sensor were 0.9705 and $0.85 \%$ respectively, while for the coupled-line sensor, they were 0.9383 and $1.30 \%$ respectively.

\section{Discussion}

Considering the differences in the samples and experimental error, the rice grain complex permittivity measurement results illustrated in Fig. 6 are comparable with those of the previous study for Lebonnet long grain rough rice at $2.45 \mathrm{GHz}$ (Nelson, 1991).

As shown in Fig. 7, the $f_{r}$ measurement data for the coupled-line sensor was more scattered than for the wide-ring sensor. This is because the former is very sensitive to the distribution of rice kernels within the coupled-line gap. Different distribution patterns of grain within the sensor's gap affect the electromagnetic wave differently and so will give different results. Besides that, the change of resonant frequency for the coupled-line senor with a $1 \%$ change in MC is higher than that of the wide-ring sensor. Hence, the coupledline sensor exhibits greater sensitivity in MC measurement than the wide-ring sensor.
The validation results show that both designed sensors have a high $R^{2}$ value and a low average error in MC prediction. This indicates that the predicted MCs for both sensors are in good agreement with the actual MC. The wide-ring sensor shows higher accuracy in MC prediction than the coupled-line sensor.

\section{Conclusion}

In this study, a microstrip wide-ring and a microstrip coupled-line sensor with low insertion loss have been presented as non-destructive sensors for rice grain MC and relative complex permittivity measurement. The complex permittivity of rice grain with different moisture levels has been measured by the designed sensors based on the resonance technique. Calibration equations for both sensors have been developed by using the relationship between the resonant frequency and MC. A comparative study of both sensors in MC measurement has been done. The analysis shows that the sensitivity of the coupled-line sensor is higher than the wide-ring sensor. However, the accuracy of the wide-ring sensor is higher than the coupled-line sensor. Validation tests show that the MC of rice grain can be predicted by widering and coupled-line sensors with average errors of 0.85 and $1.30 \%$ respectively. The proposed sensors with their open structure that operate at relatively low frequency are suitable as low cost and non-destructive sensors for rapid rice grain moisture determination. The proposed sensors are also suitable apply for other applications such as latex and soil MC determination.

\section{Acknowledgement}

The author wishes to thank Ruth Shepherd from Manchester Metropolitan University (MMU) and Pei 
Ling Leow from Universiti Teknologi Malaysia (UTM) for their valuable suggestions and proof reading to improve the readability of this paper.

\section{Funding Information}

This work is financed by the Zamalah/Institutional Scholarship and Research University Grant (GUP) under project number Q.J130000.2523.04H77 provided by the Universiti Teknologi Malaysia.

\section{Author's Contributions}

Hou Kit Mun: Design the research plan, conduct the experiment, data analysis and manuscript writing.

Kok Yeow You: Design the research plan, organize the study, coordinate the research process, data analysis and provide the technical guidance.

Mohamad Ngasri Dimon: Participated in study organization and research plan design.

\section{Ethics}

This article is original and contains unpublished material. The corresponding author confirms that all of other authors have read and approved the manuscript and no ethical issues involved.

\section{References}

Abegaonkar, M.P., R.N. Karekar and R.C. Aiyer, 1999. A microwave microstrip ring resonator as a moisture sensor for biomaterials: Application to wheat grains. Meas. Sci. Technol., 10: 195-200.

DOI: 10.1088/0957-0233/10/3/014

Ansarudin, F., Z. Abbas, J. Hassan, N.Z. Yahaya and M.A. Ismail, 2012. A simple insulated monopole sensor technique for determination of moisture content in hevea rubber latex. Meas. Sci. Rev., 12: 249-254. DOI: $10.2478 / \mathrm{v} 10048-012-0034-5$

ASABE, 2006. ASAE Standard: ASAE S352.2 Moisture Measurement-Unground Grain and Seeds. 1st Edn., American Society of Agricultural and Biological Engineers, Michigan, pp: 606.

Chang, K. and L.H. Hsieh, 2004. Microwave Ring Circuits and Related Structures. 1st Edn., John Wiley and Sons, Hoboken, ISBN-10: 047144474X, pp: 365.

Gupta, K.C., R. Garg, I. Bahl and P. Bhartia, 1996. Microstrip Lines and Slotlines. 2nd Edn., Artech House, Boston, ISBN-10: 089006766X, pp: 535.

Jafari, F., K. Khalid, W.M.D.W. Yusoff and J. Hassan, 2010. The analysis and design of multi-layer microstrip moisture sensor for rice grain. Biosyst. Eng., 106: 324-331.

DOI: 10.1016/j.biosystemseng.2010.04.005
Joshi, K.K., M.P. Abegaonkar, R.N. Karekar and R.C. Aiyer, 1997. Microstrip ring resonator as a moisture sensor for wheat grains. Proceedings of the IEEE MTT-S International Microwave Symposium, Jun. 813, IEEE Xplore Press, Denver, Colorado, USA, pp: 1679-1682. DOI: 10.1109/MWSYM.1997.596740

Jusoh, M.A., Z. Abbas, K.Y. Lee, K.Y. You and A.M. Norimi, 2011. Determination of moisture content in mortar at near relaxation frequency $17 \mathrm{GHz}$. Meas. Sci. Rev., 11: 203-206. DOI: $10.2478 / \mathrm{v} 10048-011-0031-0$

Kim, K.B., J.H. Kim, S.S. Lee and S.H. Noh, 2002. Measurement of grain moisture content using microwave attenuation at $10.5 \mathrm{GHz}$ and moisture density. IEEE Trans. Instrum. Meas., 51: 72-77. DOI: $10.1109 / 19.989904$

Kraszewski, A., 1998. Microwave aquametry-recent advances. Proceedings of the 3rd International Symposium on Humidity and Moisture, Apr. 6-8, National Physical Laboratory, London, England, pp: 187-194.

Mextorf, H., F. Daschner, M. Kent and R. Knöchel, 2012. UWB time domain transmission sensor for free-space moisture measurements. Proceedings of the IEEE MTT-S International Microwave Symposium Digest, Jun. 17-22, Montreal, QC, Canada, pp: 1-3. DOI: 10.1109/mwsym.2012.6258276

Meyer, W. and W.M. Schilz, 1981. Feasibility study of density-independent moisture measurement with microwaves. IEEE Trans. Microwave Theory Tech., 29: 732-739. DOI: 10.1109/tmtt.1981.1130436

Mohammadi, A., K.K. Khalid, P.S. Bafti and M. Homaiee, 2012. Development of microcontrollerbased microwave system to measure solid content of hevea rubber latex. Measurement, 45: 571-579. DOI: 10.1016/j.measurement.2011.10.007

Mongia, R.K., I.J. Bahl, P. Bhartia and J. Hong, 2007. RF and Microwave Coupled-line Circuits. 2nd Edn., Artech House, ISBN-10: 1596931566, pp: 549.

Nelson, S.O., 1991. Dielectric properties of agricultural products-measurements and applications. IEEE Trans. Electr. Insul., 26: 845-869. DOI: 10.1109/14.99097

Sarabandi, K. and E.S. Li, 1997. Microstrip ring resonator for soil moisture measurements. IEEE Trans. Geosci. Remote Sens., 35: 1223-1231. DOI: $10.1109 / 36.628789$

Sheen, J., 2009. Comparisons of microwave dielectric property measurements by transmission/reflection techniques and resonance techniques. Meas. Sci. Technol., 20: 1-12. DOI: $10.1088 / 0957-0233 / 20 / 4 / 042001$

Shrestha, B., J. Lee and S. Cho, 2014. Moisture Sensor Design Using Spurline RF Resonator. In: Future Information Technology, Park, J.J., Y. Pan, C.S. Kim and Y. Yang, (Eds.), Springer, Berlin, ISBN-10: 978-3-642-55037-9, pp: 111-114. 
Sumesh Sofin, R.G. and R.C. Aiyer, 2005. Measurement of dielectric constant using a Microwave Microstrip Ring Resonator (MMRR) at $10 \mathrm{GHz}$ irrespective of the type of overlay. Microwave Opt. Technol. Lett., 47: 11-14. DOI: 10.1002/mop.21066

Trabelsi, S., S.O. Nelson and M.A. Lewis, 2009. Microwave nondestructive sensing of moisture content in shelled peanuts independent of bulk density and with temperature compensation. Sens. Instrum. Food Quality Safety, 3: 114-121. DOI: $10.1007 / \mathrm{s} 11694-009-9071-7$

You, K.Y., Z. Abbas and K. Khalid, 2010a. Application of microwave moisture sensor for determination of oil palm fruit ripeness. Meas. Sci. Rev., 10: 7-14. DOI: $10.2478 / \mathrm{v} 10048-010-0002-\mathrm{x}$

You, K.Y., S.N.A. Jalani, Z. Abbas and L.L. You, 2010b. Application of bandpass filter as a sensor for rice characterization. Proceedings of the International Conference on Computer Applicatons and Industrial Electronics, Dec. 5-7, IEEE Xplore Press, KL, Malaysia, pp: 570-573.

DOI: 10.1109/ICCAIE.2010.5735145
You, K.Y., J. Salleh, Z. Abbas and L.L. You, 2011. Cylindrical slot antennas for monitoring the quality of milled rice. Proceedings of the Progress in Electromagnetics Research Symposium, Sept. 12-16, Electromagnetics Academy, Suzhou, China, pp: 1370-1373.

Zainuddin, M.F., Z. Abbas, M.H.M. Hafizi, M.A. Jusoh and M.H.H. Razali, 2013. Monopole antenna technique for determining moisture content in the Dioscorea Hispida tubers. Aust. J. Crop. Sci., 7: 1-6. 